\title{
Adiabatic Perturbation Theory and Geometric Phases for Degenerate Systems
}

\author{
Gustavo Rigolin ${ }^{1,2}$ and Gerardo Ortiz ${ }^{1}$ \\ ${ }^{1}$ Department of Physics, Indiana University, Bloomington, IN 47405, USA \\ ${ }^{2}$ Departamento de Fisica, Universidade Federal de Sao Carlos, Sao Carlos, SP 13565-905, Brazil
}

(Dated: November 6, 2018)

\begin{abstract}
We introduce an adiabatic perturbation theory for quantum systems with degenerate energy spectra. This perturbative series enables one to rigorously establish conditions for the validity of the adiabatic theorem of quantum mechanics for degenerate systems. The same formalism can be used to find non-adiabatic corrections to the non-Abelian Wilczek-Zee geometric phase. These corrections are relevant to assess the validity of the practical implementation of the concept of fractional exchange statistics. We illustrate the formalism by exactly solving a time-dependent problem and comparing its solution to the perturbative one.
\end{abstract}

PACS numbers: 03.65.Vf, 31.15.xp, 03.65.-w

Controlling the dynamics of micro and nanoscopic systems is at the heart of experimental and theoretical schemes in quantum information and nanosciences. When, in a given physical process, there is a clear separation of time scales into fast and slow that fact helps in understanding its dynamics. This is the core motivation behind the well-known adiabatic theorem of quantum mechanics for non-degenerate systems [1]. But, how fast is fast and how slow is slow? It is crucial to quantify the validity of any time-dependent approximation in a general context since what is at stake is the practical dynamical implementation of concepts such as holonomic quantum computation or the detection of fractional (adiabatic) statistics.

A main goal of the present Letter is to asses the validity of the adiabatic approximation/theorem for degenerate spectra by introducing an Adiabatic Perturbation Theory [2] for Degenerate subspaces (DAPT). DAPT is a genuine perturbative expansion in the "velocity" $v$ to drive the system from one particular configuration to another, with the perturbative corrections to the time-dependent Schrödinger equation (SE) given about the adiabatic approximation (AA). Also, DAPT is not just a contribution to the general formalism of quantum mechanics but also a practical and operational formulation to test the validity of a broad range of concepts such as non-adiabatic fractional statistics and the extent to which AA is valid for a degenerate physical system, furnishing, as a bonus, the corrections to AA. Finally, in the development of DAPT we also determine corrections to the Wilczek-Zee (WZ) non-Abelian geometric phase [3].

The dynamics of a quantum system with Hamiltonian $\mathbf{H}(t)$ is dictated by the SE

$$
\mathrm{i} \hbar v|\dot{\Psi}(s)\rangle=\mathbf{H}(s)|\Psi(s)\rangle,
$$

written in terms of the rescaled time variable $s=v t$ $(s \in[0,1])$, where $\hbar=h / 2 \pi$ with $h$ denoting Planck's constant, $T=1 / v$ is the relevant time scale of $\mathbf{H}(s)$, and the dot means $d / d s$. Consider an explicitly timedependent Hamiltonian with orthonormal eigenvectors $\left|n^{g_{n}}(s)\right\rangle$, where $g_{n}=0,1, \cdots, d_{n}-1$ labels states of the degenerate eigenspace $\mathcal{H}_{n}$ of dimension $d_{n}$ and eigenen$\operatorname{ergy} E_{n}(s), \mathbf{H}(s)\left|n^{g_{n}}(s)\right\rangle=E_{n}(s)\left|n^{g_{n}}(s)\right\rangle$, and assume that $d_{n}$ does not change during the time evolution. In general, Eq. (11) cannot be solved in closed analytic form, so one must resort to approximation schemes. Here, we are interested in developing a perturbative expansion, i.e., a DAPT, in the small parameter $v$.

Inspired by the non-degenerate perturbative scheme developed in [2], we introduce the most general ansatz state $|\Psi(s)\rangle$ describing a degenerate system

$$
|\Psi(s)\rangle=\sum_{n=0} \sum_{g_{n}=0} \mathrm{e}^{-\frac{i}{v} \omega_{n}(s)} U_{h_{n} g_{n}}^{n}(s) b_{n}(s)\left|n^{g_{n}}(s)\right\rangle,
$$

with $n=0$ representing the ground eigenspace, the dynamical phase being given by

$$
\omega_{n}(s)=\frac{1}{\hbar} \int_{0}^{s} E_{n}\left(s^{\prime}\right) \mathrm{d} s^{\prime}=v \omega_{n}(t),
$$

and $\mathbf{U}^{n}(s)$ a unitary matrix whose physical meaning will be elucidated below. (The un-summed index $h_{n}$ is associated to the initial conditions.) By replacing this ansatz into (10) and left multiplying it by $\left\langle m^{k_{m}}(s)\right|$ one gets $\dot{b}_{n}(s) U_{h_{n} g_{n}}^{n}(s)+b_{n}(s) \dot{U}_{h_{n} g_{n}}^{n}(s)+$ $\sum_{m=0} \sum_{k_{m}=0} \mathrm{e}^{-\frac{i}{v} \omega_{m n}(s)} U_{h_{m} k_{m}}^{m}(s) M_{g_{n} k_{m}}^{n m}(s) b_{m}(s)=0$, with $\omega_{m n}(s)=\omega_{m}(s)-\omega_{n}(s)$, and $M_{h_{n} g_{m}}^{n m}(s)=$ $\left\langle n^{h_{n}}(s) \mid \dot{m}^{g_{m}}(s)\right\rangle$, which for $n \neq m$ results in

$$
M_{h_{n} g_{m}}^{n m}(s)=\left\langle n^{h_{n}}(s)|\dot{\mathbf{H}}(s)| m^{g_{m}}(s)\right\rangle / \Delta_{m n}(s),
$$

with $\Delta_{m n}(s)=E_{m}(s)-E_{n}(s)$.

Let us start analyzing the limiting situation $v \rightarrow 0$, defining the degenerate adiabatic approximation (DAA). The DAA consists in neglecting the coupling between different eigenspaces $\mathcal{H}_{n}$ but not those within a given eigenspace, i.e., we set

$$
M_{g_{n} k_{m}}^{n m}(s)=\delta_{n m} M_{g_{n} k_{n}}^{n n}(s) \text { and } \dot{b}_{n}(s)=0
$$

as the degenerate adiabatic condition, implying

$$
\dot{U}_{h_{n} g_{n}}^{n}(s)+\sum_{k_{n}=0} U_{h_{n} k_{n}}^{n}(s) M_{g_{n} k_{n}}^{n n}(s)=0 \text { and }
$$




$$
\left|\Psi^{(0)}(s)\right\rangle=\sum_{n=0} \sum_{g_{n}=0} \mathrm{e}^{-\frac{\mathrm{i}}{v} \omega_{n}(s)} U_{h_{n} g_{n}}^{n}(s) b_{n}(0)\left|n^{g_{n}}(s)\right\rangle .
$$

In particular, if the system starts at the ground state $\left|0^{0}(0)\right\rangle$ we have $b_{n}(0)=\delta_{0 n}$ and $U_{h_{n} g_{n}}^{n}(0)=\delta_{h_{n} g_{n}}$ leading to

$$
\left|\Psi^{(0)}(s)\right\rangle=\sum_{g_{0}=0} \mathrm{e}^{-\frac{\mathrm{i}}{v} \omega_{0}(s)} U_{0 g_{0}}^{0}(s)\left|0^{g_{0}}(s)\right\rangle,
$$

where we have chosen $h_{0}=0$ as the initial condition $|\Psi(0)\rangle=\left|0^{0}(0)\right\rangle$. Similar expressions hold for $h_{n}=$ $1,2, \ldots, d_{n}-1$. For $n=0$ they represent the other possible initial conditions $\left|0^{1}(0)\right\rangle,\left|0^{2}(0)\right\rangle, \ldots,\left|0^{d_{0}-1}(0)\right\rangle$. Had we started in a superposition of those states the condition $U_{h_{n} g_{n}}^{n}(0)=\delta_{h_{n} g_{n}}$, or $\mathbf{U}^{n}(0)=\mathbb{1}$ using matrix notation, must be relaxed without, nevertheless, destroying the unitarity of $\mathbf{U}^{n}(s)$. Note that for definiteness, and without loss of generality, we choose $h_{n}=h_{m}=0, \forall n, m$, in the rest of this Letter. Other choices would result in different initial conditions for a fixed $\mathbf{U}^{n}(0)$ being, nevertheless, as good as long as we stick with it. As we will see below, and hinted by Eq. (4), the adiabaticity condition for degenerate systems is connected to the gap between eigenenergies associated to each degenerate eigenspace.

The adiabatic phase for degenerate systems has a geometric (WZ phase) and a dynamical component [3]. Here we prove how the WZ geometric phase corresponds to the lowest-order approximation in our DAPT. Defining $A_{h_{n} g_{m}}^{n m}(s)=\left(M_{h_{n} g_{m}}^{n m}(s)\right)^{*}$, Eq. (6) can be written as $\dot{\mathbf{U}}^{n}(s)-\mathbf{U}^{n}(s) A^{n n}(s)=0$ (the minus sign comes from $\left.M_{h_{n} g_{m}}^{n m}(s)=-\left(M_{g_{m} h_{n}}^{m n}(s)\right)^{*}\right)$. By time integration of this expression we arrive at the WZ phase

$$
\mathbf{U}^{n}(s)=\mathbf{U}^{n}(0) \hat{\mathcal{T}} \exp \left(\int_{0}^{s} \mathbf{A}^{n n}\left(s^{\prime}\right) d s^{\prime}\right),
$$

with $\hat{\mathcal{T}}$ denoting a time-ordered exponential. Under a change of basis, i.e. a gauge transformation, $\mathbf{U}^{n}(s)$ transforms unitarily after a cyclic path [3-5]. Note that in Ref. [3] the authors assumed the initial state to correspond to a single eigenvector; here we have relaxed this assumption (cf. Eq. (2)), allowing us to get a stronger condition for the validity of the DAA in its full generality, as we will see below.

We are now in a position to state the adiabatic theorem for degenerate systems: "If the Hamiltonian of a system $\mathbf{H}(t)$ changes slowly during the course of time, say from $t=0$ to $t=T$, and the system is initially prepared in an eigenstate of $\mathbf{H}$, say $\left|n^{g_{n}}(0)\right\rangle$, then it will remain in the instantaneous (snapshot) eigenspace $\mathcal{H}_{n}$ of $\mathbf{H}(t)$ during the interval $t \in[0, T] . "$ The conditions for the validity of this theorem and the determination of corrections to the DAA when it fails are what concern us in the rest of the Letter. To this end, we will need to develop a DAPT.

We want next to develop a series expansion in terms of the small adiabatic parameter $v=1 / T$, i.e., a DAPT.
As in the non-degenerate case 2], the choice of ansatz for the state $|\Psi(s)\rangle$ is crucial for its success since one needs to isolate the singular terms. This can be seen by noting that the perfect ansatz would factor out the dependence of $|\Psi(s)\rangle$ on all terms of order $\mathcal{O}\left(v^{0}\right)$ and below. In particular, terms $\mathcal{O}\left(v^{-1}\right)$ and below are the problematic ones when $v \rightarrow 0$ and should be handled with care. Since those terms come from $\mathrm{e}^{-\frac{i}{v} \omega_{n}(s)}$, while the zeroth order term comes from the WZ phase, we write down the following ansatz

$$
\begin{gathered}
|\boldsymbol{\Psi}(s)\rangle=\sum_{p=0}^{\infty} v^{p}\left|\Psi^{(p)}(s)\right\rangle, \text { where } \\
\left|\Psi^{(p)}(s)\right\rangle=\sum_{n=0} \mathrm{e}^{-\frac{i}{v} \omega_{n}(s)} \mathbf{B}_{n}^{(p)}(s)|\mathbf{n}(s)\rangle \text { and } \\
\mathbf{B}_{n}^{(p)}(s)=\sum_{m=0} \mathrm{e}^{\frac{i}{v} \omega_{n m}(s)} \mathbf{B}_{m n}^{(p)}(s) .
\end{gathered}
$$

Here $|\boldsymbol{\Psi}(s)\rangle,\left|\boldsymbol{\Psi}^{(p)}(s)\right\rangle$, and $|\mathbf{n}(s)\rangle$ are $d_{n}$-component column vectors while $\mathbf{B}_{n}^{(p)}(s)$ and $\mathbf{B}_{m n}^{(p)}(s)$ are square matrices of dimensions $d_{n} \times d_{n}$, where for definiteness we assume $d_{n}$ to represent the degree of degeneracy of the most degenerate eigenspace. For example, $|\mathbf{n}(s)\rangle^{T}=$ $\left(\left|n^{0}(s)\right\rangle,\left|n^{1}(s)\right\rangle, \cdots,\left|n^{d_{n}-1}(s)\right\rangle\right)$. Whenever the dimension of a given eigenspace $m$ is smaller than $d_{n}$, i.e., $d_{m}<d_{n}$, we pad zeros to those entries and to the corresponding ones of $\mathbf{B}_{m n}^{(p)}(s)$. We will also need the bra row vector, $\langle\mathbf{n}(s)|=\left(\left\langle n^{0}(s)\right|,\left\langle n^{1}(s)\right|, \cdots,\left\langle n^{d_{n}-1}(s)\right|\right)$. Notice that $\left\langle\left.\mathbf{n}(s)\right|^{T}\right.$ is a column vector which combined to $|\mathbf{n}(s)\rangle^{T}$ by the usual matrix multiplication rule gives $\left\langle\left.\mathbf{n}(s)\right|^{T} \mid \mathbf{n}(s)\right\rangle^{T}$, a $d_{n} \times d_{n}$ matrix. Each component of the vector $|\boldsymbol{\Psi}(s)\rangle$ has to be interpreted as corresponding to the evolution of an initial condition. Thus, if one starts at $s=0$ in the state $g_{n}=1$ of the ground eigenspace $n=0$, i.e., $\left|0^{1}(0)\right\rangle$, the relevant component describing its time evolved state is the second one, i.e., $[|\boldsymbol{\Psi}(s)\rangle]_{1}$. The reason for adopting this convention is one of notational and conceptual convenience and of mathematical simplicity in the following calculations. We will see that this is the most general ansatz accommodating an arbitrary initial state.

Another important ingredient 2] for the success of DAPT is the setting of the right initial conditions, which amounts to guaranteeing the following two constraints:

(i) The zeroth order must be the AA. In vector notation AA reads (cf. Eq. (77)),

$$
\left|\Psi^{(0)}(s)\right\rangle=\sum_{n=0} \mathrm{e}^{-\frac{i}{v} \omega_{n}(s)} b_{n}(0) \mathbf{U}^{n}(s)|\mathbf{n}(s)\rangle,
$$

implying $\mathbf{B}_{m n}^{(0)}(s)=b_{n}(0) \mathbf{U}^{n}(s) \delta_{n m}$ and $\mathbf{B}_{n}^{(0)}(s)=$ $b_{n}(0) \mathbf{U}^{n}(s)$. Moreover, if we start at $\left|0^{0}(0)\right\rangle$, implying $b_{n}(0)=\delta_{n 0}$ and $\mathbf{U}^{n}(0)=\mathbb{1}$, we reproduce Eq. (8). 
(ii) For $p \geq 1$ we must have $\left|\boldsymbol{\Psi}^{(p)}(0)\right\rangle=0$, implying $\mathbf{B}_{n n}^{(p)}(0)=-\sum_{m=0, m \neq n} \mathbf{B}_{m n}^{(p)}(0)$, and thus, $\mathbf{B}_{n}^{(p)}(0)=0$.

Having established the initial conditions one needs to insert Eqs. (10), (11), and (12), namely, $|\boldsymbol{\Psi}(s)\rangle^{T}=$ $\sum_{k, m=0} \sum_{p=0}^{\infty} v^{p} \mathrm{e}^{-\frac{i}{v} \omega_{m}(s)}|\mathbf{k}(s)\rangle^{T} \mathbf{B}_{m k}^{(p)}(s)^{T}$, into the SE (11) in vector notation, i $\hbar v|\dot{\Psi}(s)\rangle^{T}=\mathbf{H}(s)|\boldsymbol{\Psi}(s)\rangle^{T}$, to obtain the following recursive relation after left multiplying it with $\left\langle\left.\mathbf{n}(s)\right|^{T}\right.$,

$$
\frac{\mathrm{i}}{\hbar} \Delta_{m n}(s) \mathbf{B}_{m n}^{(p+1)}(s)=\dot{\mathbf{B}}_{m n}^{(p)}(s)+\sum_{k=0} \mathbf{B}_{m k}^{(p)}(s) \mathbf{M}^{k n}(s),
$$

where $\left[\mathbf{M}^{k n}(s)\right]_{h_{k} g_{n}}=\left\langle n^{g_{n}}(s) \mid \dot{k}^{h_{k}}(s)\right\rangle=M_{g_{n} h_{k}}^{n k}(s)$ (cf. Eq. (41) ) is the following square matrix

$$
\mathbf{M}^{k n}(s)=\left(\begin{array}{cccc}
M_{0,0}^{n k} & M_{1,0}^{n k} & \cdots & M_{g_{n}-1,0}^{n k} \\
M_{0,1}^{n k} & M_{1,1}^{n k} & \cdots & M_{g_{n}-1,1}^{n k} \\
\vdots & \vdots & \ddots & \vdots \\
M_{0, g_{n}-1}^{n k} & M_{1, g_{n}-1}^{n k} & \cdots & M_{g_{n}-1, g_{n}-1}^{n k}
\end{array}\right) .
$$

By construction, the zeroth order term is the DAA. The case $p=0$ and $n=m$, after using the initial constraint (i), leads to $b_{n}(0)\left(\dot{\mathbf{U}}^{n}(s)+\mathbf{U}^{n}(s) \mathbf{M}^{n n}(s)\right)=0$, thus recovering the expression for the WZ phase.

This recursive relation is the starting point for a DAPT. The first non-trivial non-adiabatic contribution is first order in $v$

$$
\begin{aligned}
& \left|\Psi^{(1)}(s)\right\rangle=\mathrm{i} \hbar \sum_{\substack{n, m=0 \\
m \neq n}} \mathrm{e}^{-\frac{\mathrm{i}}{v} \omega_{n}(s)} b_{n}(0) \mathbf{J}^{n m n}(s) \mathbf{U}^{n}(s)|\mathbf{n}(s)\rangle \\
& -\mathrm{i} \hbar \sum_{\substack{n, m=0 \\
m \neq n}} \mathrm{e}^{-\frac{\mathrm{i}}{v} \omega_{n}(s)} b_{m}(0) \frac{1 \mathbf{W}^{m n}(0) \mathbf{U}^{n}(s)}{\Delta_{n m}(0)}|\mathbf{n}(s)\rangle \\
& +\mathrm{i} \hbar \sum_{\substack{n, m=0 \\
m \neq n}} \mathrm{e}^{-\frac{\mathrm{i}}{v} \omega_{m}(s)} b_{m}(0) \frac{\mathbf{U}^{m}(s) \mathbf{M}^{m n}(s)}{\Delta_{n m}(s)}|\mathbf{n}(s)\rangle,
\end{aligned}
$$

with ${ }_{1} \mathbf{W}^{m n}(s)=\mathbf{U}^{m}(s) \mathbf{M}^{m n}(s)\left(\mathbf{U}^{n}(s)\right)^{\dagger}$,

$$
\mathbf{J}^{n m n}(s)=\int_{0}^{s} \mathrm{~d} s^{\prime}\left\{\frac{{ }_{2} \mathbf{W}^{n m n}\left(s^{\prime}\right)}{\Delta_{n m}\left(s^{\prime}\right)}\right\},
$$

and ${ }_{2} \mathbf{W}^{n m n}(s)=\mathbf{U}^{n}(s) \mathbf{M}^{n m}(s) \mathbf{M}^{m n}(s)\left(\mathbf{U}^{n}(s)\right)^{\dagger}$. Notice that $\left|\Psi^{(1)}(0)\right\rangle=0$ and if we have no degeneracy we get back to the results of [2]. In the particular case that the initial state is in the ground state subspace $|\mathbf{0}(0)\rangle$

$$
\begin{aligned}
& \left|\Psi^{(1)}(s)\right\rangle=\mathrm{i} \hbar \sum_{n=1} \mathrm{e}^{-\frac{\mathrm{i}}{v} \omega_{0}(s)} \mathbf{J}^{0 n 0}(s) \mathbf{U}^{0}(s)|\mathbf{0}(s)\rangle \\
& -\mathrm{i} \hbar \sum_{n=1} \mathrm{e}^{-\frac{\mathrm{i}}{v} \omega_{n}(s)} \frac{1 \mathbf{W}^{0 n}(0) \mathbf{U}^{n}(s)}{\Delta_{n 0}(0)}|\mathbf{n}(s)\rangle \\
& +\mathrm{i} \hbar \sum_{n=1} \mathrm{e}^{-\frac{\mathrm{i}}{v} \omega_{0}(s)} \frac{\mathbf{U}^{0}(s) \mathbf{M}^{0 n}(s)}{\Delta_{n 0}(s)}|\mathbf{n}(s)\rangle .
\end{aligned}
$$

More specifically, for an initial state $\left|0^{0}(0)\right\rangle$, the firstorder time-evolved quantum state corresponds to the first component $\left|\Psi^{(1)}(s)\right\rangle=\left[\left|\Psi^{(1)}(s)\right\rangle\right]_{0}$.

Finally, we can address the validity of the general adiabatic theorem for degenerate systems. As in the nondegenerate case, the AA is reliable as long as the contributions coming from the first order correction are small. This implies the following conditions if we start at the ground state $\left|0^{0}(0)\right\rangle$,

$$
\hbar v\left|\sum_{n=1}\left[\mathbf{J}^{0 n 0}(s) \mathbf{U}^{0}(s)\right]_{0 g_{0}}\right| \ll 1 \text {, and for all } n \geq 1 \text {, }
$$

$\hbar v\left|\frac{\left[\mathbf{U}^{0}(s) \mathbf{M}^{0 n}(s)\right]_{0 g_{n}}}{\Delta_{n 0}(s)}-\mathrm{e}^{-\frac{\mathrm{i}}{v} \omega_{n 0}(s)} \frac{\left[{ }_{1} \mathbf{W}^{0 n}(0) \mathbf{U}^{n}(s)\right]_{0 g_{n}}}{\Delta_{n 0}(0)}\right| \ll 1$,

reducing to the ones in [2] when there is no degeneracy.

To illustrate our DAPT formalism, consider a fourlevel quantum system subjected to a rotating classical magnetic field $\mathbf{B}(t)=B \mathbf{r}(t)$ of constant magnitude $B$. In spherical coordinates $\mathbf{r}(t)=(\sin \theta \cos \varphi(t), \sin \theta \sin \varphi(t)$, $\cos \theta$ ), with $0 \leq \theta \leq \pi$ and $0 \leq \varphi<2 \pi$ the polar and azimuthal angles, respectively. The Hamiltonian describing the system is [7] $\mathbf{H}(t)=\frac{\hbar}{2} b \mathbf{r}(t) \cdot \boldsymbol{\Gamma}$, with $b>0$ proportional to $B$ and $\boldsymbol{\Gamma}=\left(\Gamma_{x}, \Gamma_{y}, \Gamma_{z}\right)$ Dirac matrices satisfying the Clifford algebra $\left\{\Gamma_{i}, \Gamma_{j}\right\}=2 \delta_{i j} \mathbb{1}_{4}, j=x, y, z$. In terms of Pauli matrices $\sigma_{j}$, we choose the particular representation $\Gamma_{j}=\sigma_{x} \otimes \sigma_{j}$, so that $\left[\Gamma_{i}, \Gamma_{j}\right]=2 \mathrm{i} \epsilon_{i j k} \Pi_{k}$, with $\Pi_{j}=\mathbb{1}_{2} \otimes \sigma_{j}$, and $\epsilon_{i j k}$ the Levi-Civita symbol. Then, the snapshot eigenvectors in the basis where $\Pi_{z}$ is diagonal are $\left(n=0,1\right.$ and $\left.\alpha=e^{i \varphi(t)} \sin \theta, \beta=\cos \theta\right)$

$$
\begin{aligned}
& \left|n^{0}(t)\right\rangle=\frac{1}{\sqrt{2}}\left(\alpha^{*}|\uparrow \uparrow\rangle-\beta|\uparrow \downarrow\rangle-(-1)^{n}|\downarrow \downarrow\rangle\right), \\
& \left|n^{1}(t)\right\rangle=\frac{1}{\sqrt{2}}\left(\beta|\uparrow \uparrow\rangle+\alpha|\uparrow \downarrow\rangle-(-1)^{n}|\downarrow \uparrow\rangle\right),
\end{aligned}
$$

with two-fold degenerate, time-independent, snapshot eigenvalues $E_{0}=-(\hbar / 2) b$ and $E_{1}=(\hbar / 2) b$.

We can exactly solve the time-dependent SE (11) when $\varphi(t)=w t, w>0$ representing the frequency of the rotating magnetic field, by employing techniques similar to those developed for the single spin-1/2 problem [8 10 . Assuming that at $t=0$ the initial state is $\left|0^{0}(0)\right\rangle$, the resulting time-dependent solution, expressed in terms of the snapshot eigenvectors of $\mathbf{H}(t)$, is

$$
\begin{aligned}
& |\Psi(t)\rangle=e^{\mathrm{i} \frac{w t}{2}}\left[\frac{1+\cos \theta}{2} A_{-}(t)+\frac{1-\cos \theta}{2} A_{+}(t)\right]\left|0^{0}(t)\right\rangle \\
& +e^{-\mathrm{i} \frac{w t}{2}} \sin \theta \frac{A_{+}(t)-A_{-}(t)}{2}\left|0^{1}(t)\right\rangle \\
& +e^{\mathrm{i} \frac{w t}{2}} \sin ^{2} \theta \frac{B_{+}(t)+B_{-}(t)}{2}\left|1^{0}(t)\right\rangle \\
& +e^{-\mathrm{i} \frac{w t}{2}} \sin \theta\left[\frac{1+\cos \theta}{2} B_{-}(t)-\frac{1-\cos \theta}{2} B_{+}(t)\right]\left|1^{1}(t)\right\rangle,
\end{aligned}
$$


with $A_{ \pm}(t)=\cos \left(\Omega_{ \pm} t / 2\right)+\mathrm{i} \frac{b \pm w \cos \theta}{\Omega_{ \pm}} \sin \left(\Omega_{ \pm} t / 2\right), B_{ \pm}(t)$ $=\mathrm{i} \frac{w}{\Omega_{ \pm}} \sin \left(\Omega_{ \pm} t / 2\right)$, and $\Omega_{ \pm}^{2}=w^{2}+b^{2} \pm 2 w b \cos \theta$.

In order to develop a DAPT expansion one needs the expression for the WZ phase. For our problem it can be determined exactly $(n=0,1)$

$$
\mathbf{U}^{n}(t)=\left(\begin{array}{cc}
U_{00}^{n}(t) & U_{01}^{n}(t) \\
U_{10}^{n}(t) & U_{11}^{n}(t)
\end{array}\right)=\left(\begin{array}{cc}
z_{1} & -z_{2}^{*} \\
z_{2} & z_{1}^{*}
\end{array}\right),
$$

where $z_{1}=e^{\mathrm{i} w t / 2}\left[\cos \left(\frac{w t}{2} \cos \theta\right)-\mathrm{i} \cos \theta \sin \left(\frac{w t}{2} \cos \theta\right)\right]$ and $z_{2}=\mathrm{i} e^{\mathrm{i} w t / 2} \sin \theta \sin \left(\frac{w t}{2} \cos \theta\right)$. In this way, the zeroth order term, i.e., the DAA reads

$$
\begin{aligned}
\left|\Psi^{(0)}(t)\right\rangle & =\mathrm{e}^{-\frac{\mathrm{i}}{v} \omega_{0}(t)}\left(U_{00}^{0}(t)\left|0^{0}(t)\right\rangle+U_{01}^{0}(t)\left|0^{1}(t)\right\rangle\right) \\
& =e^{\mathrm{i} \frac{b t}{2}}\left(z_{1}\left|0^{0}(t)\right\rangle-z_{2}^{*}\left|0^{1}(t)\right\rangle\right)
\end{aligned}
$$

after replacing $\omega_{0}(s)=-b s / 2=-b v t / 2$, which is the same obtained by expanding the exact solution $|\Psi(t)\rangle$ up to zeroth order. The first non-trivial correction is first order in DAPT, where the small parameter is $v=w$. It is given by

$$
\begin{aligned}
\left|\Psi^{(1)}(t)\right\rangle & =\mathrm{i} \frac{w^{2} t}{4 b v} \sin ^{2} \theta\left|\Psi^{(0)}(t)\right\rangle+\mathrm{i} \frac{w}{b v} \sin (b t / 2) \sin \theta \\
& \times\left(z_{1} \sin \theta+z_{2} \cos \theta\right)\left|1^{0}(t)\right\rangle+\frac{w}{b v}\left[\cos (b t / 2) z_{2}^{*}\right. \\
& \left.+\mathrm{i} \sin (b t / 2) \cos \theta\left(z_{1}^{*} \sin \theta+z_{2}^{*} \cos \theta\right)\right]\left|1^{1}(t)\right\rangle,
\end{aligned}
$$

and it exactly coincides with the first order expansion of the exact solution $|\Psi(t)\rangle$. Note that in working out the expansion of the exact solution one must be aware that terms $w^{n+1} t$ are actually order $w^{n}$ since $t \propto 1 / v=1 / w$.

We should emphasize that for most time dependent Hamiltonians one is not able the get the exact solution. However, our DAPT is general enough to provide a systematic expansion in powers of $v$ about the DAA for any time dependent degenerate Hamiltonian. Furthermore, DAPT can be used to get corrections to the nonabelian WZ phase. In our example, if the first order correction to the DAA becomes relevant, the system's state can be written as $|\Psi(s)\rangle_{N}=\sqrt{P(s)}\left|\Phi^{(0)}(s)\right\rangle+$ $v \mathrm{e}^{-\mathrm{i} b t / 2} N(s)\left(c_{0}(s)\left|1^{0}(s)\right\rangle+c_{1}(s)\left|1^{1}(s)\right\rangle\right)+\mathcal{O}\left(v^{2}\right)$, where $N(s)$ normalizes the state, $P(s)=\left|\left\langle\Psi^{(0)}(s) \mid \Psi(s)\right\rangle_{N}\right|^{2}$, $\left|\Phi^{(0)}(s)\right\rangle=N(s)[1+v f(s)] / \sqrt{P(s)}\left|\Psi^{(0)}(s)\right\rangle$, and $f(s)=$ is $\sin ^{2} \theta /(4 b)$. The quantities $c_{0}(s)$ and $c_{1}(s)$ are not needed and can be obtained via Eq. (22). $P(s)$ gives the probability of measuring the first order corrected state $|\Psi(s)\rangle_{N}$ in the adiabatic state $\left|\Psi^{(0)}(s)\right\rangle$, i.e., the chances of being at ground eigenspace. The remaining terms in $\left|\Phi^{(0)}(s)\right\rangle$ determines how $\left|0^{0}(s)\right\rangle$ and $\left|0^{1}(s)\right\rangle$ are now superposed within the ground eigenspace. $\left|\Phi^{(0)}(s)\right\rangle$ is what is actually seen by any experiment trying to get the WZ phase if the first order is relevant. Keeping terms up to first order we get $\left|\Phi^{(0)}(s)\right\rangle=[1+v f(s)]\left|\Psi^{(0)}(s)\right\rangle=$ $e^{\mathrm{i} \frac{b t}{2}}\left(x_{1}\left|0^{0}(s)\right\rangle+x_{2}\left|0^{1}(s)\right\rangle\right)$, where $x_{1}=(1+v f(s)) z_{1}$ and $x_{2}=-(1+v f(s)) z_{2}^{*}$ (cf. Eq. (21)). We can repeat the previous argument but with the system starting at $\left|0^{1}(0)\right\rangle$, which gives the two remaining terms for the corrected WZ phase (cf. the second row of Eq. (20) with $n=0)$. Putting everything together and noting that $v=$ $w$ we get instead of the WZ phase $\mathbf{U}^{(0)}(t)$ the following non-abelian phase $\mathbf{V}^{(0)}(t)=\mathbf{U}^{(0)}(t)+\frac{\mathrm{i} w^{2} t \sin ^{2} \theta}{4 b} \mathbf{U}^{(0)}(t)$. This is what is experimentally seen if the first order correction is relevant. Note that $\mathbf{V}^{(0)}(t)$ is unitary up to first order and that for $v \rightarrow 0$ we recover continuously the WZ phase. Also, the previous procedure applied to the nondegenerate system of [2] gives the same correction to the Berry phase therein computed by another method.

In conclusion, we have shown a genuine adiabatic perturbation theory for Hamiltonians with degenerate spectrum (DAPT) as a series expansion about the degenerate adiabatic approximation (DAA), in terms of the "velocity" $v$ by which the system is driven away from its initial configuration. DAPT allowed us to get a general formulation to the adiabatic theorem for degenerate systems as well as corrections to the non-abelian Wilczek-Zee phase when DAA no longer holds. Finally, the key ingredients in the construction of DAPT, differentiating it from all standard perturbation theories and other approaches [6], are three fold: (a) the rescaling of the real time $t$ to $s=v t$, allowing a consistent perturbative expansion to all orders in terms of $v$; (b) the correct setting of the initial condition at $t=0$, i.e., one should recover the zeroth order not only when $v \rightarrow 0$ but also at $t=0$; and (c) the powerful vectorial ansatz, Eqs. (10)-(12), that factored out singular terms and permitted the construction of simple matrix recursive relations.

GR thanks the Brazilian agency CAPES for funding.

[1] A. Messiah, Quantum Mechanics, (North-Holland, Amsterdam, 1962), vol. 2.

[2] G. Rigolin, G. Ortiz, and V. H. Ponce, Phys. Rev. A 78, 052508 (2008).

[3] F. Wilczek and A. Zee, Phys. Rev. Lett. 52, 2111 (1984).

[4] D. Kult, J. Åberg, and E. Sjöqvist, Phys. Rev. A 74, 022106 (2006).

[5] As given in 3, 4], if we make a gauge transformation $|\tilde{\mathbf{n}}(s)\rangle=\left(\mathcal{V}^{n}(s)\right)^{\dagger}|\mathbf{n}(s)\rangle$, the two pieces of Eq. (9) transform as

$$
\begin{array}{r}
\mathbf{U}^{n}(0) \rightarrow\left(\mathcal{V}^{n}(0)\right)^{\dagger} \mathbf{U}^{n}(0) \mathcal{V}^{n}(0), \\
\hat{\mathcal{T}} \exp \left(\int_{0}^{s} \mathbf{A}^{n n}\left(s^{\prime}\right) d s^{\prime}\right) \rightarrow \\
\left(\mathcal{V}^{n}(0)\right)^{\dagger} \hat{\mathcal{T}} \exp \left(\int_{0}^{s} \mathbf{A}^{n n}\left(s^{\prime}\right) d s^{\prime}\right) \mathcal{V}^{n}(s) .
\end{array}
$$

Under the adiabatic approximation and a cyclic and closed path in the parameter space of the changing Hamiltonian [3] we have $\mathcal{V}^{n}(s=1)=\mathcal{V}^{n}(0)$. Putting 
all these pieces together

$$
\begin{array}{r}
\mathbf{U}^{n}(0) \hat{\mathcal{T}} \exp \left(\int_{0}^{s} \mathbf{A}^{n n}\left(s^{\prime}\right) d s^{\prime}\right) \rightarrow \\
\left(\mathcal{V}^{n}(0)\right)^{\dagger} \mathbf{U}^{n}(0) \hat{\mathcal{T}} \exp \left(\int_{0}^{s} \mathbf{A}^{n n}\left(s^{\prime}\right) d s^{\prime}\right) \mathcal{V}^{n}(0),
\end{array}
$$

showing that Eq. (9) transforms unitary under a gauge transformation. Note that for open paths one can have $\mathcal{V}(s=1) \neq \mathcal{V}(0)$ [4] and one must employ ideas along the line of [4] to define meaningful degenerate geometric phases.

[6] M. V. Berry, Proc. R. Soc. Lond. A 414, 31 (1987); Y. Aharonov et al., Phys. Rev. Lett. 65, 3065 (1990); E.R. Meyer et al., Phys. Rev. A 80, 062110 (2009).

[7] N. S. Biswas, Phys. Lett. B 228, 440 (1989).

[8] I. I. Rabi, N. F. Ramsey, and J. Schwinger, Rev. Mod. Phys. 26, 167 (1954).

[9] A. Bohm, Quantum Mechanics: Foundations and Applications, (Springer-Verlag, New York, 1993), p. 587.

[10] We first define the rotated state $|\tilde{\Psi}(t)\rangle=$ $\mathbf{U}^{\dagger}(t)|\Psi(t)\rangle$, which satisfies SE with Hamilto$\operatorname{nian} \tilde{\mathbf{H}}=\mathbf{U}^{\dagger}(t) \mathbf{H}(t) \mathbf{U}(t)-\mathrm{i} \hbar \mathbf{U}^{\dagger}(t) \frac{\mathrm{d} \mathbf{U}(t)}{\mathrm{d} t}$. If we choose $\mathbf{U}(t)=\mathrm{e}^{-\frac{\mathrm{i} w t}{2} \Pi_{z}}$, the resulting Hamiltonian, $\tilde{\mathbf{H}}=\frac{\hbar}{2}\left(b \Gamma_{x} \sin \theta+b \Gamma_{z} \cos \theta-w \Pi_{z}\right)$, turns out to be time independent. We have used the commutators $\left[\Pi_{z}, \Gamma_{x}\right]=\mathrm{i} 2 \Gamma_{y}$ and $\left[\Pi_{z}, \Gamma_{y}\right]=-\mathrm{i} 2 \Gamma_{x}$, leading to mathematical identity $\Gamma_{x} \cos (w t)+\Gamma_{y} \sin (w t)=\mathbf{U}(t) \Gamma_{x} \mathbf{U}^{\dagger}(t)$. The rotated frame Hamiltonian, $\tilde{\mathbf{H}}$, is no longer degenerate. Its eigenvectors are

$$
|\mu \nu(0)\rangle=\frac{\tilde{\Omega}_{\mu \nu}}{2 \sqrt{\Omega_{\eta}}}\left(|\uparrow \uparrow\rangle+b_{\mu \nu}(0)(\nu|\uparrow \downarrow\rangle-\mu|\downarrow \downarrow\rangle)-\eta|\downarrow \uparrow\rangle\right),
$$

where $\tilde{\Omega}_{\mu \nu}^{2}=\Omega_{\eta}+\mu w+\nu b \cos \theta, \Omega_{\eta}^{2}=w^{2}+b^{2}+2 \eta w$ $b \cos \theta, b_{\mu \nu}(t)=b \mathrm{e}^{\mathrm{i} w t} \sin \theta / \tilde{\Omega}_{\mu \nu}^{2}$, with $\eta=\mu \nu$ and $\mu, \nu=$ \pm ; while its eigenvalues are $\tilde{E}_{\mu \nu}=-\mu(\hbar / 2) \Omega_{\eta}$. Therefore, the exact time-dependent solution for a generic initial state $|\Psi(0)\rangle=\sum_{\mu, \nu} \tilde{a}_{\mu \nu}(0)|\mu \nu(0)\rangle$ is

$$
\begin{aligned}
|\Psi(t)\rangle & =\mathrm{e}^{-\frac{\mathrm{i} w t}{2} \Pi_{z}} \mathrm{e}^{-\frac{\mathrm{i} \tilde{\mathrm{H}} t}{\hbar}}|\Psi(0)\rangle, \\
& =\sum_{\mu, \nu} \mathrm{e}^{-\mathrm{i}\left(\frac{w}{2}+\frac{\tilde{E}_{\mu \nu}}{\hbar}\right) t} \tilde{a}_{\mu \nu}(0)|\mu \nu(t)\rangle .
\end{aligned}
$$

